\title{
Rapid Reduction in Right Atrial Size after Amplatzer Septal Occluder Placement Results in Disc Distortion and Chest Pain Necessitating Device Removal
}

\author{
Leigh Christopher Reardon, $\mathrm{MD}^{1,2^{*}}$, Jamil Anis Aboulhosn, $\mathrm{MD}^{1}$ \\ ${ }^{1}$ Department of Medicine, Ahmanson/UCLA Adult Congenital Heart Disease Center, Los Angeles, California, USA \\ ${ }^{2}$ Departement of Pediatric Cardiology, UCLA Children's Heart Center, University of California Los Angeles David Geffen School of \\ Medicine, Los Angeles, California, USA
}

\begin{abstract}
Patients with a significant shunt from a secundum atrial septal defect (ASD) undergo repair to avoid longterm complications of right ventricular failure, atrial arrhythmias, and pulmonary hypertension. Upon surgical or transcatheter ASD closure, there is an abrupt change in shunt volume, and cardiac remodeling occurs over a period of months to years. Frequently, right-sided dilation does not resolve completely. A 26-year-old man underwent device closure of a large secundum ASD and was observed to have a rapid and profound reduction in right atrial size over an 8-day period. He was asymptomatic for a week following the procedure and then began to experience worsening chest pain. He also experienced various dysrhythmias, including low atrial rhythm with pauses as well as atrial flutter. Echocardiography did not demonstrate pericardial effusion, but electrocardiogram-gated cardiac computed tomography angiography showed the right atrial disc digging into the posterior wall of the right atrium. Despite aggressive medical treatment with non-steroidal anti-inflammatory medication (i.e., opiates and steroids), the patient continued to have daily symptoms. Surgical device removal and ASD repair was carried out 29 days after device placement, which showed no gross evidence of device erosion but that the right atrial disc was compressed into the posterior wall of the right
\end{abstract}

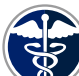

Fax +1 2037853346

E-Mail: jshd@scienceinternational.org

http://structuralheartdisease.org/ (c) 2018 Journal of Structural Heart Disease Published by Science International Corp. ISSN 2326-4004

Accessible online at: http://structuralheartdisease.org/ atrium. The patient's symptoms resolved following recovery.

Copyright $\odot 2018$ Science International Corp.

Key Words

Atrial septal defect • ASD closure • ASD device retrieval - Congenital heart disease

\section{Introduction}

We report a case of a rapid and profound change in the right atrial size of a patient who underwent device closure of a secundum atrial septal defect (ASD) using an Amplatzer septal occluder (ASO) resulting in chest pain and rhythm changes. Computed tomography angiography (CTA) showed the right atrial disc "digging" into the posterior wall of the right atrium. Upon surgical exposure of the device, the shape and position of the device was found to be distorted.

\section{Case Presentation}

A 26-year-old man with a history of a heart murmur from the age of 13 was referred to our institution for the management of a newly diagnosed secun-

* Corresponding Author:

Leigh Christopher Reardon, MD

Ahmanson/UCLA Adult Congenital Heart Disease Center and Departement of Pediatric Cardiology

University of California Los Angeles David Geffen School of Medicine

100 UCLA Medical Plaza, Suite 630E, Los Angeles, CA 90024, USA

Tel. +1 310825 9011; Fax: +1 310825 6346; E-Mail: Ireardon@mednet.ucla.edu 


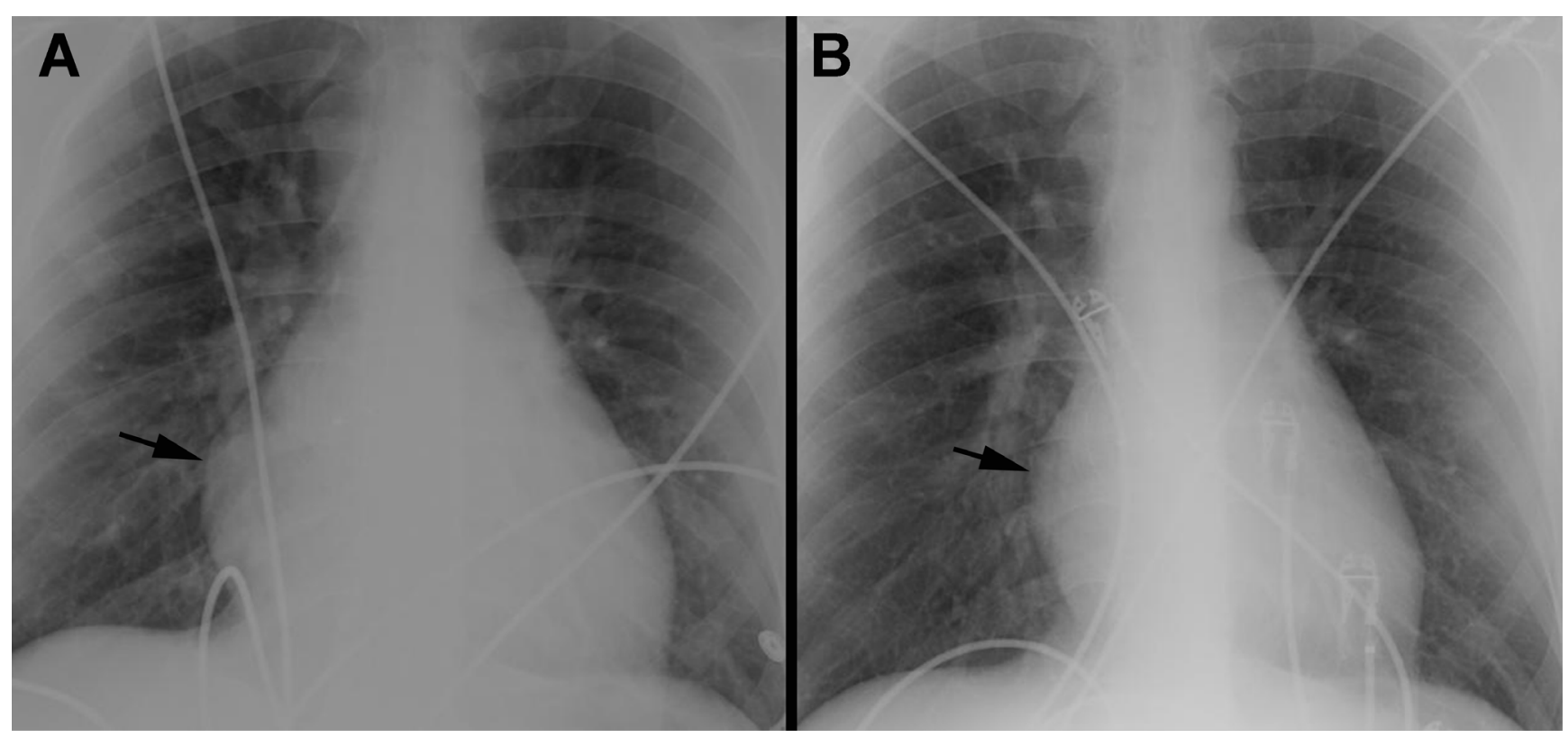

Figure 1. Chest $X$-rays demonstrating (Panel A) significant right atrial enlargement secondary to the patient's large secundum atrial septal defect (Panel $A$ ) and profound decrease in right atrial size 12 days after device placement (Panel B).

dum ASD. The patient initially presented to his primary doctor after a several-month history of decreased exercise tolerance and palpitations. On transthoracic echocardiogram, the patient was noted to have profound right atrial and right ventricular enlargement and a large ASD measuring $1.8 \times 2.2 \mathrm{~cm}$. Electrocardiogram (ECG) demonstrated a low atrial rhythm with a rate of 48 beats per minute and an Rsr' in the lead $\mathrm{V} 1$. A chest $\mathrm{X}$-ray demonstrated severe right atrial enlargement (Figure 1A).

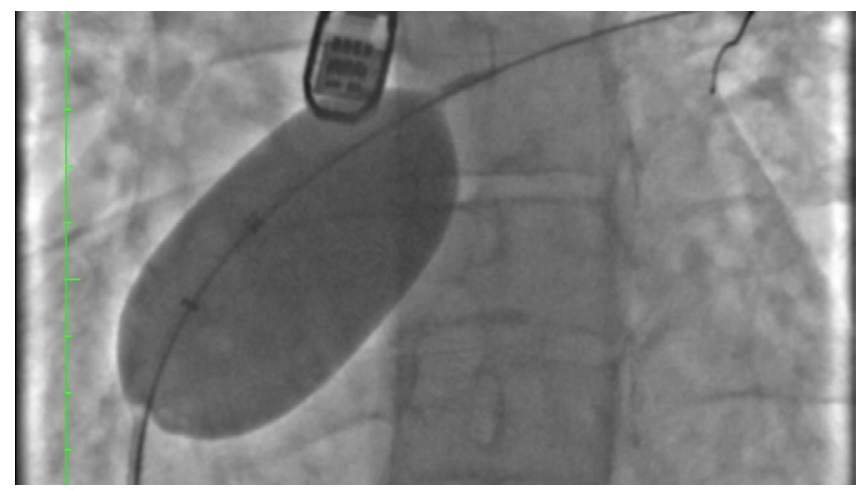

Video 1. Balloon-sizing of the atrial septal defect using a 34-mm sizing balloon. Despite stop-flow, there was no waist on the sizing balloon. View supplemental video at https://doi.org/10.12945/j. jshd.2018.036.17.vid.01.
In the catheterization lab, hemodynamic data demonstrated a normal pulmonary vascular resistance of 0.4 Wood units and a Qp:Qs of 2.7:1. Transesophageal echocardiography showed that the defect measured $30 \mathrm{~mm}$, and the stop-flow technique using a 34-mm-sized balloon did not demonstrate a waist on cineangiogram, so a 38-mm Amplatzer ASO device was chosen. An excellent result was achieved with both imaging modalities. Videos 1 and 2 show balloon-sizing of the defect and rotational angiography of the device in excellent position. The patient

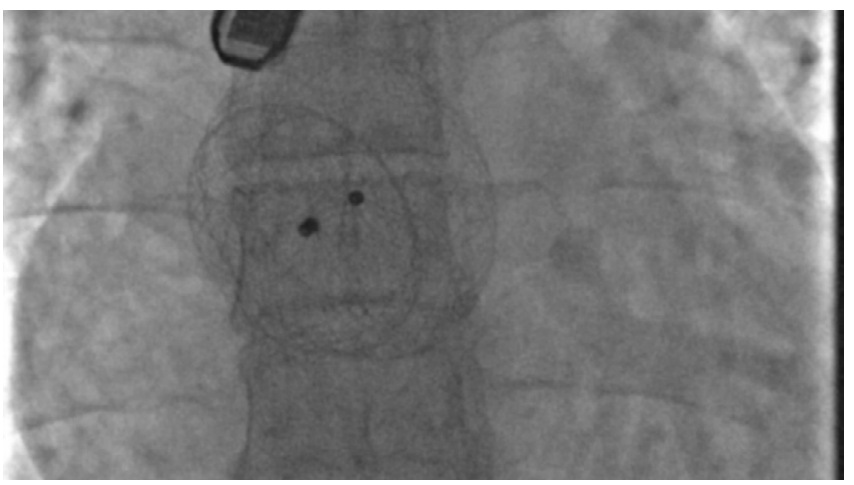

Video 2. Rotational cineangiogram after device deployment demonstrated excellent positioning of the device. View supplemental video at https://doi.org/10.12945/j.jshd.2018.036.17. vid.02. 


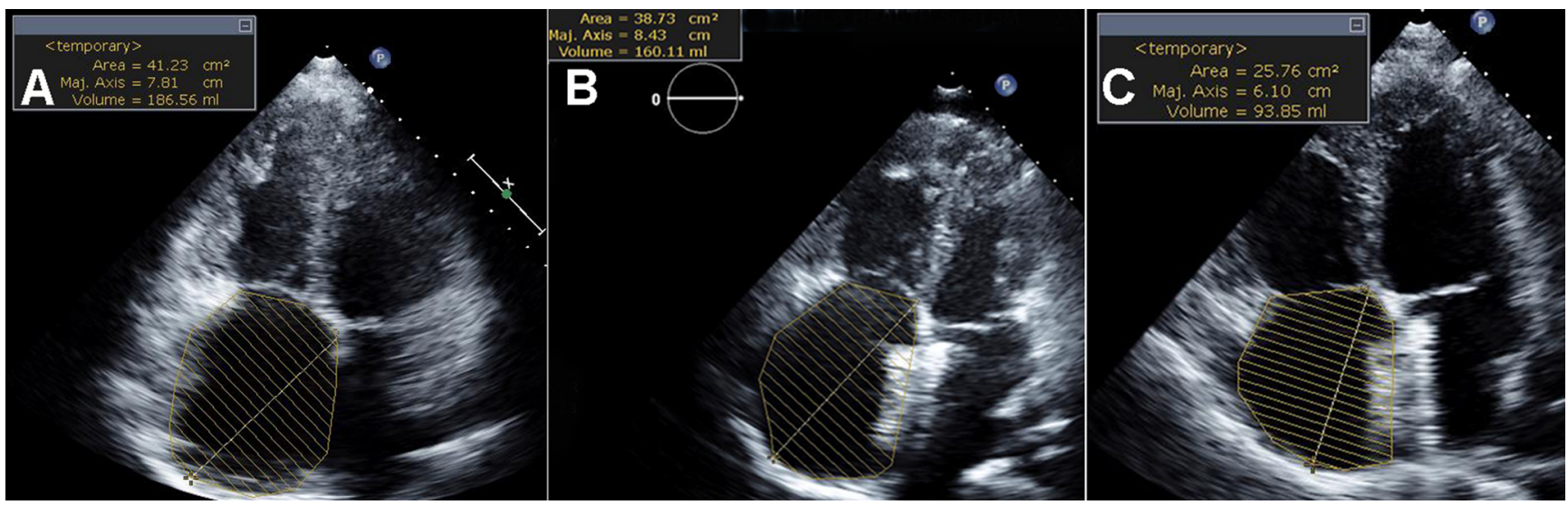

Figure 2. Echocardiogram images demonstrating right atrial volumes of $186 \mathrm{ml}$ before device closure (Panel A), $160 \mathrm{ml}$ the day after the procedure (Panel B), and $94 \mathrm{ml} 8$ days after the procedure (Panel $C$ ).

was discharged the next day after echocardiography showed excellent positioning of the device and no evidence of pericardial effusion.

The patient returned for routine follow-up 8 days later with complaints of palpitations and returned again 12 days later for an urgent visit with continued palpitations as well as chest pain and pressure. Repeat chest X-ray demonstrated a profound decrease in right atrial size (Figure 1B). Review of the patient's transthoracic echocardiogram demonstrated a decrease in right atrial volume from $186 \mathrm{ml}$ pre-procedure to $160 \mathrm{ml}$ the day after the procedure and $94 \mathrm{ml}$ 8 days after the procedure (Figure 2 ).

Despite aggressive medical treatment with non-steroidal anti-inflammatory medication (i.e., opiates and steroids), he continued to have daily symp-

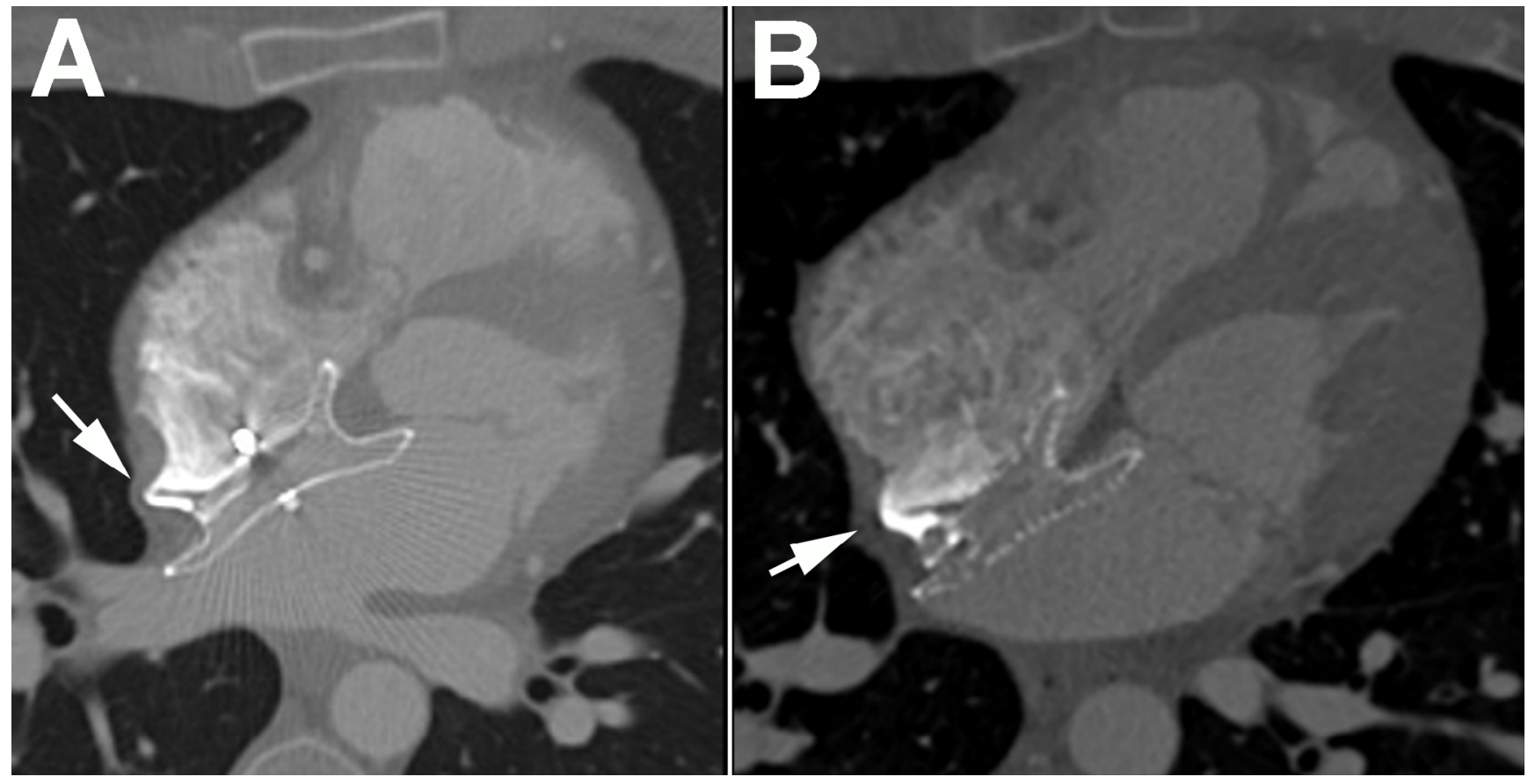

Figure 3. Computed tomography scan demonstrating the right atrial disc "digging" into the posterior wall of the right atrium and distortion of the right atrial wall (arrow). Panel A. Posterior right atrial wall distortion at the level of the pulmonary veins. Panel B. Anterior view with continued right atrial wall distortion (arrow). 
toms. ECG demonstrated a low atrial rhythm with pauses greater than $2 \mathrm{~s}$ and brief runs of atrial flutter. ECG did not demonstrate pericardial effusion, but ECG-gated cardiac CTA showed the right atrial disc "digging" into the posterior wall of the right atrium (Figure 3).

Given his persistent symptoms and rhythm disturbance, we decided that device removal and surgical closure of the ASD was the best course of action. Subsequent explantation of the device demonstrated distortion of the right atrial disk (Figure 4). The patient also had a pacemaker placed for sinus node dysfunction and has had ablations for intraatrial re-entrant tachycardia.

\section{Discussion}

Transcatheter closure of secundum ASDs with various occlusion devices has become a standard of care in most pediatric and adult patients due to greater safety and efficacy, shorter recovery duration, and decreased hospitalization time compared with surgical closure [1]. Complications are rare with transcatheter devices, and most complications that require surgical removal and ASD patch closure are secondary to device embolization [2]. Rhythm abnormalities are also reported, with supraventricular tachycardia and atrioventricular block being the most common [1].

Right-sided remodeling following ASD closure remains difficult to assess given the geometric shape of the right atrium and right ventricle. Major deformational and geometrical changes of the right ventricle are completed in 24 hours, but remodeling continues for several months to years [2]. Echocardiography data using strain rate values and tricuspid annular plane systolic excursion suggest an immediate improvement in left ventricular function and decrease in right ventricular function, likely secondary to loading conditions [3-6]. Changes in the size and shape of the atria are not well described.

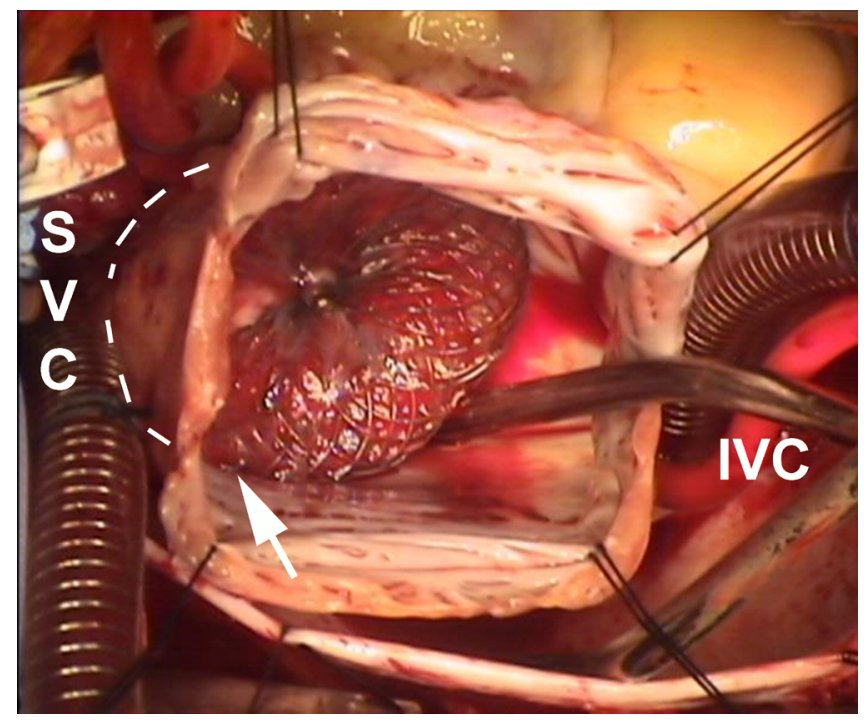

Figure 4. Surgical exposure of the right atrium revealed a distorted right atrial disc. Dashed lines demonstrate the course of the device in the right atrium. The arrow corresponds to the area of atrial distortion demonstrated on the computed tomography scan in Figure 3. SVC = superior vena cava; IVC = inferior vena cava.

In this case, chest X-ray demonstrated a profound change in right atrial size. We hypothesize that the combination of a large 38-mm ASO device and rapid change in the size of the right atrium caused a significant change in the shape of the device that provoked chest pain and rhythm disturbances. The conformational change was confirmed upon explantation of the device. Therefore, larger devices may have a higher potential to change shape and cause symptoms. Caution should be employed when utilizing larger ASD devices.

\section{Conflict of Interest}

The authors have no conflict of interest relevant to this publication.

Comment on this Article or Ask a Question 


\section{References}

1. Du ZD, Hijazi ZM, Kleinman CS, Silverman $\mathrm{NH}$, Larntz K, Amplatzer Investigators. Comparison between transcatheter and surgical closure of secundum atrial septal defect in children and adults: results of a multicenter nonrandomized trial. J Am Coll Cardiol. 2002;39:1836-1844. PMID: 12039500

2. Wu HC, Wang CC, Fu YC, Jan SL, Wei HJ, Lin $\mathrm{YK}$, et al. Surgical management for complications during closure of atrial septal defect with Amplatzer device. Acta Cardiol Sin. 2013;29:557-561. PMID: 27122757

3. Roglu E, Cakal SD, Cakal B, Dundar C, Alici G, Ozkan B, et al. Time course of right ventricular remodeling after percutaneous atrial septal defect closure: Assessment of regional deformation properties with two-dimensional strain and strain rate imaging. Echocardiography. 2013;30:324330. DOI: $10.1111 /$ echo. 12053

4. Takaya $Y$, Taniguchi M, Akagi T, Nobusada $\mathrm{S}$, Kusano $\mathrm{K}$, Ito $\mathrm{H}$, et al. Long-term effects of transcatheter closure of atrial septal defect on cardiac remodeling and exercise capacity in patients older than 40 years with a reduction in cardiopulmonary function. J Interv Cardiol. 2013;26:195-199. DOI: 10.1111/joic.12002

5. Balcı KG, Balcı MM, Aksoy MM, Yılmaz S, Aytürk $M$, Doğan $M$, et al. Remodeling process in right and left ventricle after percutaneous atrial septal defect closure in adult patients. Turk Kardiyol Dern Ars. 2015;43:250-258. PMID: 25905996

6. Jategaonkar S, Scholtz W, Schmidt $H$,
Fassbender D, Horstkotte D. Cardiac remodeling and effects on exercise capacity after interventional closure of atrial septal defects in different adult age groups. Clin Res Cardiol. 2010;99:183-91. DOI: 10.1007/ s00392-009-0105-2

Cite this article as: Reardon LC, Aboulhosn JA. Rapid Reduction in Right Atrial Size after Amplatzer Septal Occluder Placement Results in Disc Distortion and Chest Pain Necessitating Device Removal. Structural Heart Disease. 2018;4(1):28-32. DOI: https://doi. org/10.12945/j.jshd.2018.036.17 